\title{
A characterization of finite vector bundles on Gauduchon astheno-Kähler manifolds
}

\author{
Indranil Biswas and Vamsi Pritham Pingali
}

\begin{abstract}
A vector bundle on a projective variety $X$ is called finite if it satisfies a nontrivial polynomial equation with integral coefficients. Nori proved that a vector bundle $E$ on $X$ is finite if and only if there is a finite étale Galois covering $q: \widetilde{X} \longrightarrow X$ and a $\operatorname{Gal}(q)$-module $V$, such that $E$ is isomorphic to the quotient of $\widetilde{X} \times V$ by the twisted diagonal action of $\operatorname{Gal}(q)$ [Nol], [No2]. Therefore, $E$ is finite if and only if the pullback of $E$ to some finite étale Galois covering of $X$ is trivial. We prove the same statement when $X$ is a compact complex manifold admitting a Gauduchon astheno-Kähler metric.
\end{abstract}

Keywords. Finite bundles; astheno-Kähler manifolds; numerically flat bundles; Uhlenbeck-Yau theorem

2010 Mathematics Subject Classification. 32L10; 53C55; 14D21

\section{[Français]}

Titre. Une caractérisation des fibrés vectoriels finis sur les variétés asthéno-kählériennes Gauduchon. Un fibré vectoriel sur une variété projective $X$ est dit fini s'il satisfait une équation polynomiale non triviale à coefficients entiers. Nori a montré qu'un fibré vectoriel $E$ sur $X$ est fini si et seulement s'il existe un revêtement galoisien étale fini $q: \widetilde{X} \longrightarrow X$ et un $\operatorname{Gal}(q)$-module $V$ tels que $E$ soit isomorphe au quotient de $\widetilde{X} \times V$ par l'action diagonale tordue de $\operatorname{Gal}(q)$ [No1], [No2]. Par conséquent, $E$ est fini si et seulement si l'image réciproque de $E$ sur un certain revêtement galoisien fini de $X$ est triviale. Nous prouvons le même énoncé lorsque $X$ est une variété complexe compacte admettant une métrique de Gauduchon asthéno-kählérienne .

Received by the Editors on January 16, 2018, and in final form on June 22, 2018. Accepted on August 16, 2018.

\section{Indranil Biswas}

School of Mathematics, Tata Institute of fundamental research, Homi Bhabha road, Mumbai 400005, India

e-mail: indranil@math.tifr.res.in

Vamsi Pritham Pingali

Department of Mathematics, Indian Institute of Science, Bangalore 560012, India

e-mail: vamsipingali@iisc.ac.in 


\section{Contents}

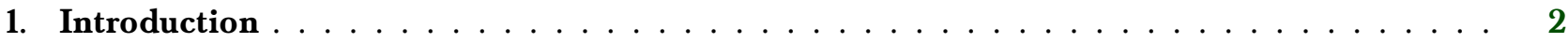

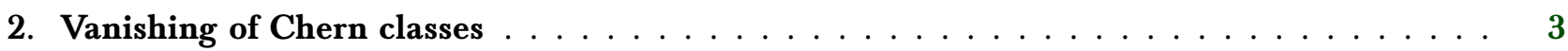

3. A characterization of numerically flat bundles on Gauduchon astheno-Kähler manifolds . 4

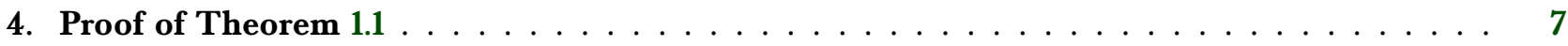

\section{Introduction}

For a polynomial $f(x)=\sum_{i=0}^{n} a_{i} x^{i}$, where $a_{i}$ are nonnegative integers, and a holomorphic vector bundle $E$ on a complex projective variety or more generally on a compact complex manifold $X$, the vector bundle

$$
\bigoplus_{i=0}^{n}\left(E^{\otimes i}\right)^{\oplus a_{i}}
$$

on $X$ is denoted by $f(E)$ (the vector bundle $E^{0}$ is defined to be the trivial line bundle $\mathcal{O}_{X}$ ). An algebraic (respectively, holomorphic) vector bundle $E$ on a projective variety (respectively, compact complex manifold) $X$ is called finite if there are two distinct polynomials $f, g$ with nonnegative integral coefficients, such that the vector bundle $f(E)$ is isomorphic to $g(E)$. It is known that a vector bundle $E$ is finite if and only if there is a finite collection of vector bundles $\left\{F_{j}\right\}_{j=1}^{m}$ and nonnegative integers $\left\{a_{i, j}\right\}_{j=1}^{m}$ such that

$$
E^{\otimes i}=\bigoplus_{j=1}^{m}\left(F_{j}\right)^{\oplus a_{i, j}}
$$

for all $i \geq 1$ [No1, p. 35, Lemma 3.1], [No2, p. 80, Lemma 3.1]. When $X$ is a compact Riemann surface, it was noted by Weil that $E$ is finite if it admits a flat connection with finite monodromy that is compatible with holomorphic structure of $E[\mathrm{We}$. Note that the condition that $E$ admits a flat connection with finite monodromy is equivalent to the condition that the pullback of $E$ to some finite étale Galois covering of $X$ is holomorphically trivial.

In a foundational work, Nori proved that a vector bundle $E$ on a complex projective variety $X$ is finite if and only if there is a finite étale Galois covering $q: \widetilde{X} \longrightarrow X$ and a $\operatorname{Gal}(q)$-module $V$, such that $E$ is isomorphic to the quotient of $\widetilde{X} \times V$ by the twisted diagonal action of $\operatorname{Gal}(q)$ [No1], [No2]. Therefore, the monodromy homomorphism of the flat connection on $E$, given by the trivial connection on $\widetilde{X} \times V \longrightarrow \widetilde{X}$, coincides with the one given by the action on $V$ of $\operatorname{Gal}(q)$ (it is a quotient of the fundamental group of $X$ ).

Let $X$ be a compact complex manifold equipped with a Hermitian form $\omega$. This pair $(X, \omega)$ is called Gauduchon if $\partial \bar{\partial} \omega^{n-1}=0$, and it is called astheno-Kähler if $\partial \bar{\partial} \omega^{n-2}=0$ [JY]. Any compact complex manifold admits a Gauduchon metric [Ga], and any complex Hermitian surface is astheno-Kähler.

Our aim here is to prove the following:

Theorem 1.1. Suppose $X$ is a compact complex manifold admitting a Hermitian metric that is both Gauduchon and astheno-Kähler. Then a holomorphic vector bundle $E$ over $X$ is finite if and only if it corresponds to a representation of a finite quotient of the fundamental group of $X$, or equivalently, if and only if $E$ admits a flat connection, compatible with its holomorphic structure, that has finite monodromy group.

Note that a flat connection with finite monodromy is a unitary connection for a suitable Hermitian structure. 
Theorem 1.1 was proved earlier in [BHS] under the assumption that $X$ is a compact Kähler manifold. The proof in [BHS] crucially used a well-known theorem of Corlette-Simpson [Si]. However, that theorem is available only in the Kähler context. Here we have been able to avoid using the Corlette-Simpson theorem.

For higher dimensional examples of Gauduchon astheno-Kähler manifolds, see [LY], [FGV], [LU], [LYZ] for instance.

\section{Vanishing of Chern classes}

In whatever follows, for a compact complex manifold $X$ we use the Bott-Chern cohomology defined as

$$
H_{B C}^{p, q}(X)=\frac{k \operatorname{er}\left(\partial: A^{p, q}(X) \rightarrow A^{p+1, q}(X)\right) \cap k \operatorname{er}\left(\bar{\partial}: A^{p, q}(X) \rightarrow A^{p, q+1}(X)\right)}{\operatorname{Im}\left(\partial \bar{\partial}\left(A^{p-1, q-1}(X)\right)\right)}
$$

where $A^{p, q}(X)$ consists of smooth $(p, q)$-forms on $X$. These groups are finite dimensional. They coincide with the Dolbeault cohomology groups for Kähler manifolds, as a consequence of the $\partial \bar{\partial}$ lemma. If $h$ is a Hermitian metric on a holomorphic vector bundle $E$ over $X$, and $\Theta_{h}$ is the curvature of its Chern connection, then for any characteristic class $\Phi$, its Chern-Weil form $\Phi\left(\Theta_{h}\right)$ is closed, and $\Phi\left(\Theta_{h_{2}}\right)-\Phi\left(\Theta_{h_{1}}\right)=$ $\partial \bar{\partial} B C\left(h_{1}, h_{2}\right)$, where $B C$ is the Bott-Chern form. Therefore, we can think of $\Phi$ as an element of the Bott-Chern cohomology (with representatives furnished by using Chern connections). Moreover, if $E=$ $S \bigoplus V$, then $[\operatorname{ch}(E)]_{B C}=[\operatorname{ch}(S)]_{B C}+[\operatorname{ch}(V)]_{B C}$ (proven by taking a direct sum metric). Likewise, we have $[\operatorname{ch}(E \otimes F)]_{B C}=[\operatorname{ch}(E)]_{B C}[\operatorname{ch}(F)]_{B C}$.

From now onwards, we denote a vector bundle $\bigoplus_{j=1}^{m}\left(F_{j}\right)^{\oplus a_{i j}}$ as $\sum_{j=1}^{m} a_{i j} F_{j}$.

Theorem 2.1. Suppose $E$ is a finite holomorphic vector bundle of rank $r$ on a compact complex manifold $X$, i.e., there exists integers $m \geq 1, a_{i, j} \geq 0, r_{j} \geq 1$ with $1 \leq j \leq m, i \in \mathbb{N}^{>0}$, and holomorphic vector bundles $V_{j}$ of ranks $r_{j}$ such that $E^{\otimes i}=\sum_{j=1}^{m} a_{i, j} V_{j}$. Then $[\operatorname{ch}(E)]_{B C}=r$.

Proof. We induct on $i$ to show that $\left[c h_{i}(E)\right]_{B C}=0$ for all $i \geq 1$.

For $i=1$, note that $\frac{n}{r}\left[c_{1}(E)\right]_{B C}=\frac{\left[c h_{1}\left(E^{\otimes n}\right)\right]_{B C}}{r^{n}}$. On the other hand, $E^{\otimes n}=\bigoplus_{j=1}^{m} a_{n, j} V_{j}$. So,

$$
\frac{\left[c h_{1}\left(E^{\otimes n}\right)\right]_{B C}}{r^{n}}=\sum_{j=1}^{m} a_{n, j} \frac{\left[c_{1}\left(V_{j}\right)\right]_{B C}}{r^{n}}=\sum_{j=1}^{m} r_{j} a_{n, j} \frac{\left[c_{1}\left(V_{j}\right)\right]_{B C} / r_{j}}{r^{n}} .
$$

Since $r^{n}=\sum_{j=1}^{k} r_{j} a_{n, j}$, it follows that $\sum_{j=1}^{m} a_{n, j} \frac{\left[c_{1}\left(V_{j}\right)\right]_{B C}}{r^{n}}$ lies in a bounded subset of $H_{B C}^{1,1}(X, \mathbb{C})$; indeed it lies in the convex subset generated by $\left\{\left[c_{1}\left(V_{j}\right) / r_{j}\right]_{B C}\right\}_{j=1}^{k}$. However, $\frac{n}{r}\left[c_{1}(E)\right]_{B C}$ is unbounded as $n$ varies if $\left[c_{1}(E)\right]_{B C}$ is nonzero. Therefore, it follows that $\left[c_{1}(E)\right]_{B C}=0$.

Suppose $\left[\operatorname{ch}_{i}(E)\right]_{B C}=0$ for all $1 \leq i \leq k-1$. Then,

$$
\frac{\left[c h_{k}\left(E^{\otimes n}\right)\right]_{B C}}{r^{n}}=\frac{n}{r}\left[\operatorname{ch}_{k}(E)\right]_{B C},
$$

and hence $\frac{\left[c h_{k}\left(E^{\otimes n}\right)\right]_{B C}}{r^{n}}$ is unbounded as $n$ varies if $\left[c h_{k}(E)\right]_{B C}$ is nonzero. On the other hand, we have

$$
\sum_{j=1}^{m} a_{n, j} \frac{\left[c h_{k}\left(V_{j}\right)\right]_{B C}}{r^{n}}=\sum_{j=1}^{m} r_{j} a_{n, j} \frac{\left[c h_{k}\left(V_{j}\right)\right]_{B C} / r_{j}}{r^{n}},
$$


so $\sum_{j=1}^{m} a_{n, j} \frac{\left[c h_{k}\left(V_{j}\right)\right]_{B C}}{r^{n}}$ lies in the convex subset of $H_{B C}^{1,1}(X, \mathbb{C})$ generated by $\left\{\left[c h_{k}\left(V_{j}\right) / r_{j}\right]_{B C}\right\}_{j=1}^{k}$ and is hence bounded. Thus, as before, $\left[\operatorname{ch}_{k}(E)\right]_{B C}=0$.

Take a finite vector bundle $E$ and vector bundles $V_{j}, 1 \leq j \leq m$, as in Theorem 2.1. We may assume that

(1) each $V_{j}$ is indecomposable (if $V_{j}=\bigoplus_{k=1}^{\ell} W_{k}$ with each $W_{k}$ indecomposable, then replace $V_{j}$ by $\left.\left\{W_{k}\right\}_{k=1}^{\ell}\right)$,

(2) the isomorphism classes of $V_{j}$ are distinct, and

(3) for each $1 \leq j \leq m$, there is some $j^{\prime} \geq 1$ such that $a_{j^{\prime}, j}$ in Theorem 2.1 is nonzero (if $a_{i, j}=0$ for all $i$ then discard $V_{j}$ ).

Lemma 2.2. Each of the above vector bundles $V_{j}, 1 \leq j \leq m$, is finite.

Proof. Take any $V_{j}$. Take any $k \geq 1$ such that $a_{k, j} \geq 1$. Then for every $n \geq 1$, the vector bundle $V_{j}^{\otimes n}$ is a direct summand of

$$
E^{\otimes k n}=\sum_{\ell=1}^{m} a_{k n, \ell} V_{\ell}
$$

If $W$ be a holomorphic vector bundle on $X$, and

$$
\sum_{\alpha=1}^{a} A_{\alpha}=W=\sum_{\alpha=1}^{b} B_{\alpha}
$$

where all $A_{\alpha}$ and $B_{\alpha}$ are indecomposable holomorphic vector bundles, then a theorem of Atiyah says that

- $a=b$, and

- there is a permutation $\sigma$ of $\{1, \cdots, a\}$ such that the holomorphic vector bundle $A_{\alpha}$ is holomorphically isomorphic to $B_{\sigma(\alpha)}$ for all $\alpha \in\{1, \cdots, a\}$.

(See [At, p. 315, Theorem 3].) In view of this theorem, it follows from (2.1) that the direct summand $V_{j}^{\otimes n}$ is a direct sum of copies of $V_{\ell}, 1 \leq \ell \leq m$. Therefore, $V_{i}$ is finite.

\section{A characterization of numerically flat bundles on Gauduchon astheno- Kähler manifolds}

We recall the definition of a nef vector bundle as in [DPS]. A line bundle $L$ over a compact complex manifold $M$, equipped with a Hermitian metric $\omega$, is called nef if given any real number $\epsilon>0$, there is a smooth Hermitian metric $h$ on $L$ such that $\Theta\left(h_{\epsilon}\right)+\epsilon \omega$ is a nonnegative Hermitian form, where $\Theta\left(h_{\epsilon}\right)$ is the Chern curvature form. This definition actually does not depend on the choice of $\omega$. A holomorphic vector bundle $V$ on $M$ is called nef if the tautological line bundle $\mathcal{O}_{\mathbb{P}(V)}(1)$ on $\mathbb{P}(V)$ is nef. A holomorphic vector bundle $V$ is called numerically flat if both $V$ and $V^{*}$ are nef.

We note that Lemma 2.4 of [BHS], which was obtained as a corollary of Theorem 1.12 of [DPS], is stated in the Kähler set-up, because [BHS] is entirely in the Kähler set-up. However, Theorem 1.12 of [DPS] is proved for compact complex manifolds. Hence Lemma 2.4 of [BHS] remains valid for compact complex manifolds.

Lemma 3.1. ([BHS, Lemma 2.4]) If $E$ is finite, then $E$ is numerically flat. 
Proof. Since $E$ is finite, there are finitely many indecomposable bundles $V_{1}, \ldots, V_{k}$ such that $E^{\otimes i}=$ $\sum_{j=1}^{k} a_{i, j} V_{j} \forall i \geq 1$. The symmetric product $\operatorname{Sym}^{i}(E)$ is a direct summand of the vector bundle $E^{\otimes i}$. Hence from [At, p. 315, Theorem 3] (quoted in Lemma 2.2) it follows that

$$
\operatorname{Sym}^{i}(E)=\bigoplus_{j=1}^{k} a_{i, j}^{\prime} V_{j}
$$

where $a_{i, j}^{\prime} \leq a_{i, j}$ are nonnegative integers. For each $i \geq 1$, fix an isomorphism of $\operatorname{Sym}^{i}(E)$ with $\bigoplus_{j=1}^{k} a_{i, j}^{\prime} V_{j}$.

Now fix smooth Hermitian structures $g_{j}$ on $V_{j}$. This produces a Hermitian structure on any direct sum of copies of $V_{j}$ such that the direct sum decomposition is orthogonal. Consequently, we get a Hermitian structure $h_{i}$ on $\operatorname{Sym}^{i}(E)$ using the above isomorphism.

Let $\Theta_{j}$ be the curvature of the Chern connection on the Hermitian vector bundle $\left(V, g_{j}\right)$. So the curvature $\Theta_{i}$ of the Chern connection for $\left(\operatorname{Sym}^{i}(E), h_{i}\right)$ satisfies the equation

$$
\Theta_{i}=\bigoplus_{j=1}^{k} a_{i, j}^{\prime} \Theta_{i}
$$

(coefficients correspond to direct sum).

According to Theorem 1.12 of [DPS], the vector bundle $E$ is nef if for every $\epsilon>0$ and $m \geq m_{0}(\epsilon)$ we have

$$
\Theta_{m} \geq-m \epsilon \omega \otimes \operatorname{Id}_{\operatorname{Sym}^{m}(E)}
$$

in the sense of Griffiths. It is straight-forward to check that this condition is satisfied. Hence the finite vector bundle $E$ is nef.

We now prove an analogue of Theorem 1.18 of Demailly-Peternell-Schneider [DPS] in our special case of astheno-Kähler manifolds.

Theorem 3.2. Suppose $(X, \omega)$ is a compact complex manifold equipped with a Hermitian metric $\omega$ that is both, Gauduchon $\left(\partial \bar{\partial} \omega^{n-1}=0\right)$, as well as astheno-Kähler $\left(\partial \bar{\partial} \omega^{n-2}=0\right)$. Suppose a holomorphic vector bundle $E$ of rank $r$ over $X$ satisfies $\left[c_{2}(E)\right]_{B C}=0$. Then it is numerically flat if and only if the following three conditions hold:

(1) E is $\omega$-semi-stable,

(2) there is a Jordan-Hölder filtration

$$
\{0\}=E_{0} \subset E_{1} \subset \ldots \subset E_{p-1} \subset E_{p}=E
$$

consisting of subbundles, and

(3) for all $1 \leq i \leq p$, the $\omega$-stable bundle $E_{i} / E_{i-1}$ is Hermitian flat, i.e., given by an irreducible unitary representation of $\pi_{1}(X)$ in $\mathrm{U}\left(\operatorname{rank}\left(E_{i} / E_{i-1}\right)\right)$.

Remark 3.3. It can be shown that $\left[c_{1}(E)\right]_{B C}=\left[c_{1}(\operatorname{det}(E))\right]_{B C}=0$ whenever $E$ is numerically flat. Indeed, applying Proposition 1.14 (ii) of [DPS] to the determinant representation of $E$ and $E^{*}$ we see that $\operatorname{det}(E)$ is numerically flat. Now Corollary 1.5 of [DPS] implies that $\operatorname{det}(E)$ is Hermitian flat and hence $\left[c_{1}(\operatorname{det}(E))\right]_{B C}=0$. We are grateful to the anonymous referee for pointing this out. 
Proof of Theorem 3.2. A Hermitian flat bundle is clearly numerically flat. Now from [DPS, Proposition 1.15(ii)] it follows that any holomorphic vector bundle admitting a filtration of holomorphic subbundles such that each successive quotient admits a flat Hermitian structure is also numerically flat. Consequently, $E$ is numerically flat if the three conditions in the theorem hold.

We prove the converse below. Assume that $E$ is numerically flat.

Our proof inducts on the rank of $E$. Note that if $S \subset E$ is a holomorphic subbundle then the vector bundle $E / S$ is nef because $E$ is nef [DPS, p. 308, Proposition 1.15(i)]. If $S$ is nef, then applying [DPS, p. 308, Proposition 1.15(iii)] to the exact sequence

$$
0 \longrightarrow(E / S)^{*} \longrightarrow E^{*} \longrightarrow S^{*} \longrightarrow 0
$$

we conclude that $(E / S)^{*}$ is nef. Therefore, $E / S$ is numerically flat if $S$ is so. These observations are crucial for the induction step.

Since $\omega$ is assumed to be Gauduchon, we see that for every holomorphic line bundle $L$,

$$
\int c_{1}(L) \wedge \omega^{n-1} \in \mathbb{R}
$$

is independent of the choice of Chern connection on $L$. Moreover, because $\omega$ is astheno-Kähler, for any $(2,2)$ Chern-Weil form $\Phi$, the integral

$$
\int \Phi \wedge \omega^{n-2} \in \mathbb{R}
$$

is independent of the choice of Chern connection, i.e., independent of the Bott-Chern representative.

Let $\mathcal{W}$ be a coherent analytic subsheaf of $\mathcal{O}(E)$ such that the quotient $\mathcal{O}(E) / \mathcal{W}$ is torsion-free. This implies that $\mathcal{W}$ is reflexive [Ko, p. 153, Proposition 4.13]. The rank of $\mathcal{W}$ will be denoted by $q_{w}$. Then the arguments of Step 1 in the proof of Theorem 1.18 in [DPS] go through to show that $\int_{X}\left[c_{1}(\mathcal{W})\right]_{B C} \wedge \omega^{n-1} \leq 0$. Now assume that

$$
\int_{X}\left[c_{1}(\mathcal{W})\right]_{B C} \wedge \omega^{n-1}=0
$$

Then we have the following:

- $\operatorname{det}(\mathcal{W})$, which is a holomorphic line bundle, is Hermitian flat,

- $\left[c_{1}(\mathcal{W})\right]_{B C}=0$, and

- the homomorphism $\operatorname{det}(\mathcal{W}) \longrightarrow \Lambda^{q_{w}} E$ has no nontrivial zeroes (that is, $\operatorname{det}(\mathcal{W})$ is a line subbundle of $\left.\Lambda^{q_{w}} E\right)$.

Since $\operatorname{det}(\mathcal{W})$ is a line subbundle of $\Lambda^{q_{w}} E$, it follows that $\mathcal{W}$ is a subbundle of $E$ (recall that $\mathcal{O}(E) / \mathcal{W}$ is torsion-free); see [DPS, Lemma 1.20].

Now suppose $\mathcal{F} \subset \mathcal{O}(E)$ is a minimal rank $q>0$ subsheaf such that $\mathcal{O}(E) / \mathcal{F}$ is torsion-free and

$$
\int_{X}\left[c_{1}(\mathcal{F})\right]_{B C} \wedge \omega^{n-1}=0
$$

As noted before, $\mathcal{F}$ is a holomorphic subbundle of $E$ satisfying

$$
\left[c_{1}(\mathcal{F})\right]_{B C}=0 .
$$

We also note that $\mathcal{F}$ is stable, because it is of minimal rank satisfying (3.2). Therefore, the Kobayashi-Lübke inequality says that

$$
\int_{X}\left(2 q \cdot\left[c_{2}(\mathcal{F})\right]_{B C}-(q-1)\left[c_{1}(\mathcal{F})^{2}\right]_{B C}\right) \wedge \omega^{n-2} \geq 0
$$


see [LYZ, p. 107]. We will now show that in (3.4) the equality holds.

It was noted earlier that $\mathcal{W}$ is a subbundle of $E$ if it satisfies the condition in (3.2). Consequently, we get a filtration of holomorphic subbundles

$$
0=\mathcal{F}_{0} \subset \mathcal{F}=: \mathcal{F}_{1} \subset \mathcal{F}_{2} \subset \cdots \subset \mathcal{F}_{m-1} \subset \mathcal{F}_{m}=E
$$

such that each successive quotient $\mathcal{F}_{i} / \mathcal{F}_{i-1}, 1 \leq i \leq m$, satisfies the condition

$$
\left[c_{1}\left(\mathcal{F}_{i} / \mathcal{F}_{i-1}\right)\right]_{B C}=0,
$$

and $\mathcal{F}_{i} / \mathcal{F}_{i-1}$ is stable. Indeed, $E / \mathcal{F}_{1}$ is a bundle satisfying condition (3.5) (by the cup product formula for the Chern classes in Bott-Chern cohomology, equation (3.3), and the fact that $\left[c_{1}(E)\right]_{B C}=0$ by remark 3.3). The arguments in step 2 of the proof of Theorem 1.18 in [DPS] show that it is numerically flat. Therefore, we may induct on the rank of $E$ to get the above filtration.

Let $q_{i}$ be the rank of $\mathcal{F}_{i} / \mathcal{F}_{i-1}$. We again have the Kobayashi-Lübke inequality

$$
\int_{X}\left(2 q_{i} \cdot\left[c_{2}\left(\mathcal{F}_{i} / \mathcal{F}_{i-1}\right)\right]_{B C}-\left(q_{i}-1\right)\left[c_{1}\left(\mathcal{F}_{i} / \mathcal{F}_{i-1}\right)^{2}\right]_{B C}\right) \wedge \omega^{n-2} \geq 0
$$

for every $1 \leq i \leq m$. Since $\left[c_{1}\left(\mathcal{F}_{i} / \mathcal{F}_{i-1}\right)\right]_{B C}=0$, we see that

$$
\int_{X}\left[c_{2}\left(\mathcal{F}_{i} / \mathcal{F}_{i-1}\right)\right]_{B C} \wedge \omega^{n-2} \geq 0
$$

By the cup product formula (and $\left[c_{1}(E)^{2}\right]_{B C}=0$ ) we see that

$$
\int_{X}\left[c_{2}(E)\right]_{B C} \wedge \omega^{n-2} \geq 0
$$

Recall that $\left[c_{2}(E)\right]_{B C}=0$ by assumption; therefore, we see that equality holds in all of the above inequalities.

Since $\mathcal{F}$ is $\omega$-stable, by Li-Yau and Lübke-Teleman's generalization ([LY], [LT]) of the Uhlenbeck-Yau theorem [UY], it follows that $\mathcal{F}$ admits a Hermite-Einstein metric. Since the inequality in (3.4) is an equality, the Hermite-Einstein metric on $\mathcal{F}$ is flat and hence has vanishing Chern classes in the Bott-Chern cohomology. Now we take the quotient $E / \mathcal{F}$ and use the induction hypothesis. Note that the remarks made earlier show that the quotient is numerically flat, and the cup product formula shows that it has vanishing $\left[c_{2}\right]_{B C}$.

\section{Proof of Theorem 1.1}

Any representation $W$ of a finite group $G$ has the property that there are two distinct polynomials $f$ and $g$ with nonnegative integral coefficients such that the two $G$-modules $f(W)$ and $g(W)$ are isomorphic. Indeed, this follows from the following two facts

- any finite dimension $G$-module is a direct sum of irreducible $G$-modules, and

- there are only finitely many isomorphism classes of irreducible G-modules.

Therefore, if a holomorphic vector bundle $E$ on $X$ is given by a linear representation of $\pi_{1}(X)$ with finite image, then $E$ is finite.

To prove the converse, let $E$ be a finite vector bundle over a compact complex manifold $X$ admitting a Gauduchon astheno-Kähler metric. Therefore, there exists integers $m \geq 1, a_{i j} \geq 0, r_{j} \geq 1$ with $1 \leq j \leq m$, $i \in \mathbb{N}^{>0}$, and holomorphic vector bundles $V_{j}$ of ranks $r_{j}$ such that

$$
E^{\otimes i}=\sum_{j=1}^{m} a_{i j} V_{j} .
$$


We may assume that the collection of vector bundles $\left\{V_{j}\right\}_{j=1}^{m}$ satisfies the three conditions stated prior to Lemma 2.2.

From Theorem 2.1, Lemma 3.1, and Theorem 3.2 we know that $E$ has a filtration of holomorphic subbundles

$$
\{0\}=E_{0} \subset E_{1} \subset \ldots \subset E_{p-1} \subset E_{p}=E
$$

such that for all $1 \leq i \leq p$, the quotient bundle $E_{i} / E_{i-1}$ is given by an irreducible unitary representation of $\pi_{1}(X)$.

Lemma 4.1. For every $1 \leq i \leq p$, the vector bundle $E_{i} / E_{i-1}$ in (4.2) is finite.

Proof. From Lemma 2.2, Theorem 2.1, Theorem 3.2, and Lemma 3.1 we see that each $V_{j}$ admits a filtration of holomorphic subbundles such that each successive quotient is given by an irreducible unitary representation of $\pi_{1}(X)$. Fix such a Jordan-Hölder filtration

$$
\{0\}=V_{j, 0} \subset V_{j, 1} \subset \ldots \subset V_{j, p_{j}-1} \subset V_{j, p_{j}}=V_{j}
$$

for each $1 \leq j \leq m$, and consider the collection of vector bundles

$$
\left\{V_{j, i} / V_{j, i-1}\right\}, \quad 1 \leq i \leq p_{j}, 1 \leq i \leq m .
$$

Every vector bundle in the collection in (4.4) is given by an irreducible unitary representation of $\pi_{1}(X)$.

We will show that every vector bundle in the collection in (4.4) is finite. More precisely, we will show that for any $V_{j, i} / V_{j, i-1}$ in (4.4), and any integer $\alpha \geq 1$, the tensor power $\left(V_{j, i} / V_{j, i-1}\right)^{\alpha}$ is a direct sum of copies of vector bundles from the collection in (4.4).

To prove this, note that the filtration of $V_{j}$ in (4.3) produces a holomorphic filtration of subbundles

$$
\{0\}=U_{j}^{0} \subset U_{j}^{1} \subset U_{j}^{2} \cdots \subset V_{j}^{\alpha}
$$

of $V_{j}^{\alpha}$. The filtration $V_{j}^{\alpha}$ in (4.5) has the following properties:

(1) each successive quotient is of the form

$$
\bigotimes_{\beta=1}^{p_{j}}\left(V_{j, \beta} / V_{j, \beta-1}\right)^{\otimes n_{\beta}},
$$

where $n_{\beta} \geq 0$ and $\sum_{\beta=1}^{p_{j}} n_{\beta}=\alpha$, and

(2) $\left(V_{j, i} / V_{j, i-1}\right)^{\alpha}$ is one of the successive quotients.

Therefore, each successive quotient in (4.5) is a direct sum of stable vector bundles of degree zero, because it admits a flat unitary connection given by the flat unitary connections on $V_{j, \beta} / V_{j, \beta-1}, 1 \leq \beta \leq p_{j}$. It should be clarified that each successive quotient in (4.5) is polystable, but need not be stable, because the flat unitary connection on $V_{j, \beta} / V_{j, \beta-1}$ need not be irreducible.

On the other hand, from the proof of Lemma 2.2 we know that

$$
V_{j}^{\alpha}=\sum_{\gamma=1}^{m} e_{\gamma, j} V_{\gamma} .
$$

Fix an isomorphism of $V_{j}^{\alpha}$ with the direct $\operatorname{sum} \sum_{\gamma=1}^{m} e_{\gamma, j} V_{\gamma}$. Then fix an ordering of these direct summands of $V_{j}^{\alpha}$. Now using the filtrations of $V_{j}, 1 \leq j \leq m$, in (4.3), and the above ordering of the direct summands of $V_{j}^{\alpha}$, we get a filtration

$$
\{0\}=W_{j}^{0} \subset W_{j}^{1} \subset \cdots \subset W_{j}^{\ell_{1}-1} \subset W_{j}^{\ell_{1}}=V_{j}^{\alpha}
$$


such that each successive quotient $W_{j}^{b} / W_{j}^{b-1}, 1 \leq b \leq \ell_{1}$, is a vector bundle from the collection in (4.4).

We recall that for a Jordan-Hölder filtration of a semi-stable holomorphic vector bundle $F$ with successive quotients $Q_{i}$ reflexive, the isomorphism class of the coherent sheaf $\bigoplus_{i} Q_{i}$ is unique (see [BTT, Proposition 2.1 (p. 998) and Proposition A.2 (p. 1019)]). Consider the two filtrations of subbundles of $V_{j}^{\alpha}$ in (4.5) and (4.6). For the filtration in (4.5), each successive quotient is a direct sum of stable vector bundles of degree zero, while for the filtration in (4.6), each successive quotient is a stable vector bundle of degree zero. Therefore, from Theorem 3 of [At] and the above result of [BTT] we conclude that each successive quotient for the filtration in (4.5) is a direct sum of some successive quotients for the filtration in (4.6). We noted above that all the successive quotients $W_{j}^{b} / W_{j}^{b-1}, 1 \leq b \leq \ell_{1}$, in (4.6) are vector bundles from the collection in (4.4). Therefore, we now conclude that each successive quotient for the filtration in (4.5) is a direct sum of copies of vector bundles from the collection in (4.4). In particular, the vector bundle $\left(V_{j, i} / V_{j, i-1}\right)^{\alpha}$ is a direct sum of copies of vector bundles from the collection in (4.4). Therefore, the vector bundle $\left(V_{j, i} / V_{j, i-1}\right)^{\alpha}$ is finite.

Next, from (4.1) we have

$$
E=\sum_{j=1}^{m} a_{1 j} V_{j}
$$

Fix an isomorphism of $E$ with $\sum_{j=1}^{m} a_{1 j} V_{j}$. Then fix an ordering of these direct summands of $E$. Now using the filtrations of $V_{j}, 1 \leq j \leq m$, in (4.3), and the above ordering of the direct summands of $E$, we get a filtration

$$
\{0\}=W^{0} \subset W^{1} \subset \cdots \subset W^{\ell_{0}-1} \subset W^{\ell_{0}}=E
$$

such that each successive quotient $W^{b} / W^{b-1}, 1 \leq b \leq \ell_{0}$, is a vector bundle from the collection in (4.4).

Consider the two filtrations of subbundles of $E$ in (4.2) and (4.7). For both of them, the successive quotients are stable of degree zero. Therefore, from the above mentioned result of [BTT], namely [BTT, Proposition 2.1 (p. 998) and Proposition A.2 (p. 1019)], we conclude that the graded sheaves for filtrations in (4.2) and (4.7) are isomorphic. We noted above that all the successive quotients $W^{b} / W^{b-1}, 1 \leq b \leq \ell_{0}$, in (4.7) are vector bundles from the collection in (4.4). Therefore, using Theorem 3 of [At] we now conclude that each successive quotient $E_{i} / E_{i-1}, 1 \leq i \leq p$, in (4.2) is a vector bundle from the collection in (4.4).

We have already proved that every vector bundle in (4.4) is finite. Hence each successive quotient $E_{i} / E_{i-1}, 1 \leq i \leq p$, in (4.2) is a finite vector bundle.

The following lemma show that $E_{i} / E_{i-1}$ can be trivialized by pulling back to some finite unramified covering of $X$.

Lemma 4.2. If $X$ is a compact complex manifold admitting a Gauduchon astheno-Kähler metric, and $E$ is a finite holomorphic vector bundle on $X$, then there is a finite unramified connected Galois cover $\pi: Y \longrightarrow X$ such that the pull back $\pi^{*} E$ admits a filtration of holomorphic subbundles with the property that each successive quotient is a holomorphically trivial vector bundle.

Proof. Lemma 4.1 shows that each stable vector bundle $E_{i} / E_{i-1}$ in (4.2) is finite. Theorem 3.2 shows that the $E_{i} / E_{i-1}$ has an irreducible unitary flat connection. We claim that the Hermite-Einstein flat connection on $E_{i} / E_{i-1}$ actually has finite monodromy. Indeed, this follows from Proposition 2.8 of [BHS]. The monodromy representation of the Hermite-Einstein flat connection on $E_{i} / E_{i-1}$ produces a finite unramified Galois covering of $X$.

Now take $Y$ to be a connected component of the fiber product of the $p$ unramified Galois coverings of $X$ given by $\left\{E_{i} / E_{i-1}\right\}_{i=1}^{p}$. The pullback to $Y$ of the filtration in (4.2) has the required property.

The following lemma shows that the holomorphic vector bundle $\pi^{*} E$ in Lemma 4.2 is trivial. 
Lemma 4.3. If $E$ is a finite holomorphic vector bundle of rank $r$ on a compact complex manifold $Y$ such that there is a fltration of holomorphic subbundles

$$
0=E_{0} \subset E_{1} \subset \cdots \subset E_{r-1} \subset E_{r}=E
$$

satisfying the conditions that $\operatorname{rank}\left(E_{i}\right)=i$, and the holomorphic line bundle $E_{i} / E_{i-1}$ is trivial for all $1 \leq i \leq r$. Then E is holomorphically trivial.

Proof. Since $E$ is finite, there are finitely many indecomposable holomorphic vector bundles $V_{1}, \cdots, V_{m}$ such that

$$
E^{\otimes i}=\sum_{j=1}^{m} c_{i, j} V_{j}
$$

for all $i \geq 1$, where $c_{i, j}$ are nonnegative integers. By Lemma 2.2 the $V_{i}$ can be chosen to be finite bundles themselves. Given that $E_{i} / E_{i-1}$ is trivial for all $1 \leq i \leq r$, each vector bundle $E^{\otimes i}$ admits a filtration of holomorphic subbundles such that each successive quotient of it is the trivial line bundle. Akin to the proof of Lemma 4.1, by the (weak) uniqueness of the graded object of a Jordan-Hölder filtration in the sense of Proposition 2.1 of [BTT] and from Theorem 3 of [At] we see that the $V_{i}$ also admit such filtrations.

When $r=1$, there is nothing to prove. Next we consider the case of rank two. Assume that

(1) $r=\operatorname{rank}(E)=2$, and

(2) $E$ is not trivial.

Therefore, $E$ fits in a short exact sequence of holomorphic vector bundles

$$
0 \longrightarrow E_{1}=\mathcal{O}_{Y} \longrightarrow E \stackrel{\gamma}{\longrightarrow} \mathcal{O}_{Y}=E / E_{1} \longrightarrow 0,
$$

which does not split holomorphically, because $E$ is not trivial.

For any integer $i \geq 1$, let $\operatorname{Sym}^{i}(E)$ denote the $i$-th symmetric power of $E$; it is a direct summand of $E^{\otimes i}$, meaning $\operatorname{Sym}^{i}(E) \subset E^{\otimes i}$, and there is a holomorphic subbundle $S_{i} \subset E^{\otimes i}$ such that the natural homomorphism

$$
\operatorname{Sym}^{i}(E) \oplus S_{i} \longrightarrow E^{\otimes i}
$$

is an isomorphism. From this it follows that there are finitely many vector bundles $\left\{V_{j}^{\prime}\right\}_{j=1}^{m^{\prime}}$ from the collection $V_{1}, \ldots, V_{m}$ such that

$$
\operatorname{Sym}^{i}(E)=\sum_{j=1}^{m^{\prime}} c_{i, j}^{\prime} V_{j}^{\prime}
$$

for all $i \geq 1$, where $c_{i, j}^{\prime}$ are nonnegative integers. Indeed, since the $V_{j}$ are indecomposable, this follows from Theorem 3 of [At]. Recall that each $V_{j}^{\prime}$ admits a filtration of holomorphic subbundles such that each successive quotient of it is the trivial line bundle. In particular, we have

$$
\operatorname{dim} H^{0}\left(Y, V_{j}^{\prime}\right) \geq 1, \quad \forall 1 \leq j \leq m^{\prime} .
$$

We will now show that

$$
\operatorname{dim} H^{0}\left(Y, \operatorname{Sym}^{n}(E)\right)=1, \quad \forall n \geq 1 .
$$

Let

$$
\tau \in H^{1}\left(Y, \operatorname{Hom}\left(\mathcal{O}_{Y}, \mathcal{O}_{Y}\right)\right)=H^{1}\left(Y, \mathcal{O}_{Y}\right)
$$

be the extension class for the short exact sequence in (4.10). We have

$$
\tau \neq 0
$$


because (4.10) does not split holomorphically. Let

$$
0 \longrightarrow H^{0}\left(Y, \mathcal{O}_{Y}\right) \stackrel{\alpha}{\longrightarrow} H^{0}(Y, E) \longrightarrow H^{0}\left(Y, \mathcal{O}_{Y}\right) \stackrel{\varphi}{\longrightarrow} H^{1}\left(Y, \mathcal{O}_{Y}\right)
$$

be the long exact sequence of cohomologies for the short exact sequence in (4.10). Since the homomorphism $\varphi$ in (4.14) sends $1 \in H^{0}\left(Y, \mathcal{O}_{Y}\right)=\mathbb{C}$ to $\tau \in H^{1}\left(Y, \mathcal{O}_{Y}\right)$ (this follows from the construction of the extension class), we conclude that $\alpha$ in (4.14) is an isomorphism. Therefore, (4.13) holds for $m=1$.

Now we will prove (4.13) using induction on $m$.

Suppose (4.13) holds for all $n \leq n_{0}-1$, with $n_{0} \geq 2$. To prove (4.13) for $n=n_{0}$, consider the short exact sequence of holomorphic vector bundles

$$
0 \longrightarrow \operatorname{Sym}^{n_{0}-1}(E) \longrightarrow \operatorname{Sym}^{n_{0}}(E) \stackrel{\gamma^{\otimes n_{0}}}{\longrightarrow} \operatorname{Sym}^{n_{0}}\left(\mathcal{O}_{Y}\right)=\mathcal{O}_{Y} \longrightarrow 0,
$$

where $\gamma$ is the projection in (4.10). The above inclusion of $\operatorname{Sym}^{n_{0}-1}(E)$ in $\operatorname{Sym}^{n_{0}}(E)$ is given by the natural inclusion $E^{\otimes\left(n_{0}-1\right)} \otimes E_{1} \hookrightarrow E^{\otimes\left(n_{0}-1\right)} \otimes E=E^{\otimes n_{0}}$ (see (4.10)). Let

$$
0 \longrightarrow H^{0}\left(Y, \operatorname{Sym}^{n_{0}-1}(E)\right) \stackrel{\beta}{\longrightarrow} H^{0}\left(Y, \operatorname{Sym}^{n_{0}}(E)\right) \longrightarrow H^{0}\left(Y, \mathcal{O}_{Y}\right) \stackrel{\varphi^{\prime}}{\longrightarrow} H^{1}\left(Y, \operatorname{Sym}^{n_{0}-1}(E)\right)
$$

be the long exact sequence of cohomologies associated to the above short exact sequence of vector bundles. Now consider the composition

$$
\left(\gamma^{\otimes\left(n_{0}-1\right)}\right)_{*} \circ \varphi^{\prime}: H^{0}\left(Y, \mathcal{O}_{Y}\right) \longrightarrow H^{1}\left(Y, \operatorname{Sym}^{n_{0}-1}\left(\mathcal{O}_{Y}\right)\right)=H^{1}\left(Y, \mathcal{O}_{Y}\right),
$$

where $\varphi^{\prime}$ is the homomorphism in (4.15), and

$$
\gamma^{\otimes\left(n_{0}-1\right)}: \operatorname{Sym}^{n_{0}-1}(U) \longrightarrow \operatorname{Sym}^{n_{0}-1}\left(\mathcal{O}_{Y}\right)=\mathcal{O}_{Y}
$$

is the homomorphism given by $\gamma$ in (4.10). This $\left(\gamma^{\otimes\left(n_{0}-1\right)}\right)_{*} \circ \varphi^{\prime}$ sends $1 \in H^{0}\left(Y, \mathcal{O}_{Y}\right)=\mathbb{C}$ to $\left(n_{0}-1\right) \tau \in$ $H^{1}\left(Y, \mathcal{O}_{Y}\right)$, which is nonzero because $\tau \neq 0$. So, the homomorphism $\left(\gamma^{\otimes\left(n_{0}-1\right)}\right)_{*} \circ \varphi^{\prime}$ is injective, implying that $\varphi^{\prime}$ is injective. Consequently, the homomorphism $\beta$ in (4.15) is an isomorphism. This completes the proof of (4.13).

From (4.11), (4.13) and (4.12) it follows that $\sum_{j=1}^{m^{\prime}} c_{i, j}^{\prime} \leq 1$ for all $i \geq 0$. This implies that

$$
i+1=\operatorname{rank}\left(\operatorname{Sym}^{i}(E)\right) \leq \max _{1 \leq j \leq m^{\prime}} \operatorname{rank}\left(V_{j}^{\prime}\right), \quad \forall i \geq 1 .
$$

In view of this contradiction we conclude that the lemma holds for $r=2$.

We will now prove the general case.

Assume that

(1) $r=\operatorname{rank}(E)>2$, and

(2) $E$ is not trivial.

We will show that there is a subbundle $F \subset E$ with $2 \leq \operatorname{rank}(F)=s \leq r=\operatorname{rank}(E)$, and a filtration of holomorphic subbundles

$$
0=F_{0} \subset F_{1} \subset \cdots \subset F_{s-1} \subset F_{s}=F,
$$

satisfying the following conditions:

(1) $\operatorname{rank}\left(F_{i}\right)=i$ for all $1 \leq i \leq s$,

(2) the holomorphic line bundles $F_{i} / F_{i-1}, 1 \leq i \leq s$, are trivial, and

(3) the rank two holomorphic vector bundle $F / F_{s-2}$ is not trivial. 
To construct a filtration as in (4.16), take $s$ to be the smallest positive integer such that the vector bundle $E_{s}$ in (4.8) is nontrivial. So $s \geq 2$, and the vector bundle $E_{s-1}$ is isomorphic to $\mathcal{O}_{Y}^{\oplus(s-1)}$. Moreover, $E_{s}$ fits in a short exact sequence of holomorphic vector bundles

$$
0 \longrightarrow \mathcal{O}_{Y}^{\oplus(s-1)} \longrightarrow E_{s} \longrightarrow \mathcal{O}_{Y}=E_{s} / E_{s-1} \longrightarrow 0 .
$$

The corresponding extension class

$$
\delta:=\left(\delta_{1}, \cdots, \delta_{s-1}\right) \in H^{1}\left(Y, \operatorname{Hom}\left(\mathcal{O}_{Y}, \mathcal{O}_{Y}^{\oplus(s-1)}\right)\right)=H^{1}\left(Y, \mathcal{O}_{Y}\right)^{\oplus(s-1)}
$$

is nonzero, where $\delta_{i} \in H^{1}\left(Y, \mathcal{O}_{Y}\right)$, because $E_{s}$ is nontrivial, implying that the above short exact sequence of vector bundles does not split holomorphically. Choose an integer $j_{0} \in[1, s-1]$ such that $\delta_{j_{0}} \neq 0$. Now in (4.16), take

- $F_{i}=E_{i}$ for all $0 \leq i \leq j_{0}-1$ (see (4.8)),

- $F_{i}=E_{i+1}$ for all $j_{0} \leq i \leq s-2$,

- $F_{s-1}=E_{j_{0}}$, and

- $F=F_{s}=E_{s}$.

This filtration clearly satisfies all the three conditions.

Note that for all $i \geq 1$, the symmetric product $\operatorname{Sym}^{i}(F)$ is a subbundle of $\operatorname{Sym}^{i}(E)$, and $\operatorname{Sym}^{i}\left(F / F_{s-2}\right)$ is a quotient of $\operatorname{Sym}^{i}(F)$. Since $F / F_{s-2}$ is a nontrivial extension of $\mathcal{O}_{Y}$ by $\mathcal{O}_{Y}$, we have

$$
\operatorname{dim} H^{0}\left(Y, \operatorname{Sym}^{i}\left(F / F_{s-2}\right)\right)=1, \quad \forall i \geq 1,
$$

as proved in (4.13); note that the proof of (4.13) only uses that the extension in (4.10) is nontrivial, and does not use that the rank two vector bundle is finite.

Since $\operatorname{Sym}^{i}\left(F / F_{s-2}\right)$ admit a filtration of holomorphic subbundles such that each successive quotient of it is the trivial line bundle, from (4.17) it follows that $\operatorname{Sym}^{i}\left(F / F_{s-2}\right)$ is indecomposable for all $i \geq 1$. Consequently, the indecomposable components (direct summands) of $\operatorname{Sym}^{i}(E)$ are of arbitrarily large rank as $i$ goes to infinity. Since $\operatorname{Sym}^{i}(E)$ is a direct summand of $E^{\otimes i}$, the indecomposable components of $E^{\otimes i}$ are also of arbitrarily large rank as $i$ goes to infinity. But from (4.9) it follows that these ranks are bounded by $\max _{1 \leq j \leq m} \operatorname{rank}\left(V_{j}\right)$.

In view of the above contradiction, the lemma is proved.

The vector bundle $\pi^{*} E$ in Lemma 4.2 is finite because $E$ is finite. So from Lemma 4.3 we know that $\pi^{*} E$ is holomorphically trivial. Let $D$ be a connection on $\pi^{*} E$ given by a trivialization of it (this connection does not depend on the choice of the trivialization). Let $\Gamma:=\operatorname{Gal}(\pi)$ be the Galois group for the Galois covering $\pi$. For any $g \in \Gamma$, the vector bundle $g^{*} \pi^{*} E$ is trivial, and any isomorphism between $\pi^{*} E$ and $g^{*} \pi^{*} E$ takes the connection $D$ on $\pi^{*} E$ to the connection $g^{*} D$ on $g^{*} \pi^{*} E$. Therefore, $D$ descends to a connection on $E$; this descended connection will be denoted by $D_{E}$. We note that $D_{E}$ is flat because $D$ is so. The monodromy representation for $D_{E}$ clearly factors through the quotient $\pi_{1}(X) \longrightarrow \Gamma$. Therefore, $E$ admits a flat connection with finite monodromy group. This completes the proof of Theorem 1.1.

Acknowledgements. The authors are sincerely grateful to the anonymous referee for pointing out subtle errors in our earlier proofs and giving other useful feedback. The work of the second author (Pingali) was partially supported by SERB grant No. ECR/2016/001356. The second author also thanks the Infosys foundation for supporting him through the Infosys young investigator grant. The first author is supported by a J. C. Bose Fellowship. 


\section{References}

[At] M. F. Atiyah, On the Krull-Schmidt theorem with application to sheaves, Bull. Soc. Math. Fr. 84 (1956), 307-317. MR-0086358

[BHS] I. Biswas, Y. Holla, and G. Schumacher, On a characterization of finite vector bundles as vector bundles admitting a flat connection with finite monodromy group, Proc. Amer. Math. Soc. 128 (2000), no. 12, 3661-3669. MR-1695096

[BTT] N. Buchdahl, A. Teleman, and M. Toma, A continuity theorem for families of sheaves on complex surfaces, J. Topol. 10 (2017), no. 4, 995-1028. MR-3743066

[DPS] J.P. Demailly, T. Peternell, and M. Schneider, Compact complex manifolds with numerically effective tangent bundles, J. Algebraic Geom. 3 (1994), no. 2, 295-346. MR-1257325

[FGV] A. Fino, G. Grantcharov and L. Vezzoni, Astheno-Kähler and balanced structures on fibrations, Int. Math. Res. Not., to appear. arXiv:1608.06743

[Ga] P. Gauduchon, La 1-forme de torsion d'une variété hermitienne compacte, Math. Ann. 267 (1984), no. 4, 495-518. MR-0742896

[JY] J.Jost and S.-T. Yau, A nonlinear elliptic system for maps from Hermitian to Riemannian manifolds and rigidity theorems in Hermitian geometry, Acta Math. 170 (1993), no. 2, 221-254. MR-1226528

[Ko] S. Kobayashi, Differential geometry of complex vector bundles. Publications of the Mathematical Society of Japan, vol. 15; Kanô Memorial Lectures, vol. 5. Princeton University Press, Princeton, NJ; Princeton University Press, Princeton, NJ, 1987. MR-0909698

[LU] A. Latorre and L. Ugarte, On non-Kähler compact complex manifolds with balanced and astheno-Kähler metrics, C. R. Math. Acad. Sci. Paris 355 (2017), no. 1, 90-93. MR-3590290

[LY] J. Li and S.-T. Yau, Hermitian-Yang-Mills connection on non-Kähler manifolds. In: Mathematical aspects of string theory (San Diego, Calif., 1986), pp. 560-573, Adv. Ser. Math. Phys., vol. 1, World Sci. Publishing, Singapore, 1987. MR-0915839

[LYZ] J. Li, S.-T. Yau, and F. Zheng, On projectively flat Hermitian manifolds, Comm. Anal. Geom. 2 (1994), no. 1, 103-109. MR-1312680

[LT] M. Lübke and A. Teleman, The Kobayashi-Hitchin correspondence, World Scientific Publishing Co., Inc., River Edge, NJ, 1995. MR-1370660

[Nol] M. V. Nori, On the representations of the fundamental group, Compositio Math. 33 (1976), no. 1, 29-41. MR-0417179

[No2] M. V. Nori, The fundamental group-scheme, Proc. Indian Acad. Sci. Math. Sci. 91 (1982), no. 2, 73-122. MR-0682517

[Si] C. Simpson, Higgs bundles and local systems, Inst. Hautes Études Sci. Publ. Math. 75 (1992), 5-95. MR-1179076

[UY] K. Uhlenbeck and S.-T. Yau, On the existence of Hermitian-Yang-Mills connections in stable vector bundles. Frontiers of the mathematical sciences: 1985 (New York, 1985). Comm. Pure Appl. Math. 39 (1986), no. S, suppl., S257-S293. MR-0861491

[We] A. Weil, Généralisation des fonctions abélinnes, J. Math. Pures Appl. 17 (1938), 47-87. 